\title{
Original thinking and ambiguous figure reversal rates
}

\author{
HOLGER KLINTMAN \\ Lund University, Lund, Sweden
}

(Bruce O. Bergum, sponsor)

This study examined the relationship between figure reversal rates in the Necker cube and levels of original thinking as assessed by two parallel versions of the alternate-uses test. Forty-nine subjects were divided into three groups on the basis of their originality test scores. In the Necker cube, the total number of reversals experienced over a 2-min viewing period was used as a response measure. A significant difference was found between high and low originality scores, the former displaying higher reversal rates than the latter. The results support the hypothesis of a positive relationship between perceptual instability and creativity/originality.

Figure reversal rate has been well documented in the literature as an indicator of perceptual stability. The reversal phenomenon is easy to produce and shows a relatively high degree of retest reliability (J. E. Bergum \& B. O. Bergum, 1980; Mason, Kaszor, \& Bourassa, 1973). For instance, perceptual instability (high reversal rates) is indicated in the Necker cube test by a subject's tendency to experience shifts from one perspective to another, whereas perceptual stability (low reversal rates) is an expression of resistance to such changes. Explanations for the reversal phenomenon have tended to fall into two general categories, one emphasizing automatic and passive processes of neural fatigue (e.g., Attneave, 1971; Kohler, 1960; Orbach, Erlich, \& Heath, 1963), the other stressing a more active process of problem solving or of shifts in selective attention (Ammons, 1954; Gregory, 1970, 1974; Smith, Imparato, \& Exner, 1968).

Systematic differences between individuals with regard to figure reversal rates have recently led researchers to ask whether interpretation of part of the variation could be generalized to behavior at a higher cognitive level, including personality and individual associative style (B. O. Bergum \& Flamm, 1975;Klintman, 1978; Mason et al., 1973). Specifically, one might hypothesize that a high reversal rate would be correlated with superior associative fluency and with relative ease in switching from one mental perspective to another, whereas low reversal rates would characterize subjects with a slower and more rigid associative functioning. Given the accumulated evidence for a neural satiating mechanism (Long \& Toppino, 1981), this would imply a system in which the function of such a mechanism is

This study was supported by a grant from the Swedish Humanistic and Social Science Research Council. The author's mailing address is: Department of Psychology, Lund University, 22101 Lund, Sweden. to some extent modulated by higher level decisional factors.

Following this line of reasoning, it would seem reasonable to expect a possible relationship between perceptual instability and such capacities as general creativity and original thinking. Thus, in a recent study, B. O. Bergum and J. E. Bergum (1979) demonstrated that persons in visually creative occupations display significantly higher levels of perceptual instability than do people engaged in less creative activities. In a subsequent study (J. E. Bergum \& B. O. Bergum, 1979), they found a positive relationship between self-perceived general creativity and originality, on the one hand, and ambiguous figure reversal rate, on the other. Given earlier reported findings regarding the validity of selfrated creativity (B. O. Bergum, 1975), these results imply a possible link between creative capacity and perceptual instability.

In the present study, one aspect of creativitynamely, the level of original thinking-was selected, and the actual test performance of a group of subjects on this variable was assessed, the hypothesis being that high scorers on original thinking would display higher ambiguous figure reversal rates than would low scorers.

To test this hypothesis, an experiment was conducted in which a random sample of undergraduate psychology students was tested in terms of Necker cube reversal rates and given two parallel versions of the alternateuses test.

\section{METHOD}

\section{Subjects}

Forty-nine (20 male and 29 female) undergraduate students of psychology, aged 18 to 25 years, served as the subjects.

\section{Apparatus}

A Necker cube was presented in the form of a line figure drawn in black on white cardboard, and it was viewed by the 
subject from a distance of $50 \mathrm{~cm}$. The side dimensions of the front and back faces of the cube were both $28 \mathrm{~mm}$, with the back face above and $7 \mathrm{~mm}$ to the right of the front face.

The Necker cube, covered with a sheet of paper, was placed on the table in front of the subject and uncovered by the experimenter at the start of the viewing period.

The level of original thinking was measured with two parallel paper-and-pencil versions of the alternate-uses test, the stimulus words being the Swedish equivalents of "BRICK" and "A POUND OF IRON."

\section{Procedure}

Each subject was given a 2 -min trial with the Necker cube. The subject was instructed to fixate a black dot in the center of the cube and say "now" each time, and only when, he or she clearly perceived an apparent change in the orientation of the cube. Using a digital chronometer, the experimenter scored the cumulated time for each reversal.

After the Necker cube trial, the subject was given the alternateuses test. The subject was instructed to list as many uses to which the stimulus object could be put as he or she could think of. It was emphasized that, although the uses should have some logical basis, they might well be unusual, original, or unexpected. The two test versions were taken in succession, each with a time limit of $3 \mathrm{~min}$.

\section{RESULTS}

The responses obtained on the alternate-uses test were broken down into a number of categories with regard to common meaning. For instance, one response to "BRICK" might be "to use in a wall" and another "to build a house with," which would place them both in the same category of "construction material." Classification was carried out independently by two judges, resulting in 20 categories for one version and 28 for the other. For a given subject, each response was then scored in terms of the inverse of its group frequency, that is, of the total number of responses in that category. Thus, a response given by 20 subjects would be scored $1 / 20$, and a response given by only one subject would be scored 1. The median of these scores was used as a measure of relative originality of that subject.

The 49 subjects were split into three subgroups on the basis of their originality scores in the two test versions: one low group (below group median in both tests), one medium group (below median in one test and above in the other), and one high group (above median in both tests).

Table 1 gives a summary of mean reversal frequencies and standard deviations for the three subgroups. The values for the total sample are also given. As can be

Table 1

Mean Necker Cube Reversal Frequencies and Standard Deviations in 2 Min

\begin{tabular}{lcrr}
\hline $\begin{array}{c}\text { Originality } \\
\text { Score }\end{array}$ & $\mathrm{n}$ & Mean & \multicolumn{1}{c}{ SD } \\
\hline High & 15 & 16.60 & 14.40 \\
Medium & 21 & 7.90 & 5.71 \\
Low & 13 & 6.38 & 5.27 \\
Total & 49 & 9.96 & 10.00 \\
\hline
\end{tabular}

seen, the mean number of reversals was greatest for the high-originality group (16.60) and lowest for the low group (6.38).

A one-way analysis of variance with three factor levels indicated significant differences in reversal rates of the three independent subgroups $[\mathrm{F}(2,46)=5.41$, $\mathrm{p}<.01$ ]. Inspection of Table 1 suggests a maximum differentiation point somewhere between the combined low and medium groups and the high group $(\mathrm{p}<.001$, $t$ test).

A similar analysis using response frequencies, rather than originality scores, yielded no significant differences.

\section{DISCUSSION}

The observed relationship between level of originality and figure reversal rate confirms the hypothesis that a given capacity for original thinking is associated with a roughly predictable level of perceptual instability. Since originality in thinking is frequently characteristic of creators, the results support earlier studies indicating a possible link between perceptual instability and creative potential (B. O. Bergum \& J. E. Bergum, 1979; J. E. Bergum \& B. O. Bergum, 1979).

To clarify the logic of the observed relationship, let us consider briefly what is specifically required of a subject if he or she is to obtain a high originality score in the alternate-uses test. In looking for an acceptable response to the stimulus word, the subject may make use of associative links between the object and various applications well established in his or her past experience with that object. Much of this experience will inevitably be shared with other group members, since the objects are functional parts of their everyday lives. This strategy will result in predominantly high-frequency responses.

Alternatively, the subject may solve the task by actually creating new such links, that is, may indicate uses that were not "there before." These responses tend to be idiosyncratic and shared by few, if any, other subjects (low-frequency responses).

It should be noted that the two groups did not differ in terms of response-frequency in the alternate-uses test. Therefore, to obtain a high score on originality, the subject must (1) give one or more low-frequency responses and (2) give relatively few high-frequency responses. Assuming that any given subject is capable of giving high-frequency responses, to become a high scorer, he or she must then not only be able to come up with unusual solutions, but must also to some extent actively avoid high-frequency responses and favor the low-frequency ones.

In contrast, low scores may have lesser capacity than high scorers to find new and original solutions-or they may use a response strategy that constantly gives priority to "ordinary" solutions before the more unusual ones.

In the present results, the readiness of the high scorer to free him- or herself from old and well-established conceptual patterns and to construct new ones is reflected perceptually in high reversal rates. In such subjects, a figural perspective once established is quickly abandoned and a new one formed. In contrast, the characteristic resistance of the low scorer to forming, or accepting, new solutions is reflected in low reversal rates: The existing figural perspective is favored and restructuring is slow.

\section{REFERENCES}

Ammons, R. B. (1954). Experiential factors in visual form perception: I. Review and formulation of problems. Journal of Genetic Psychology, 84, 3-25.

Attnenve, F. (1971). Multistability in perception. Scientific American, 225(6), 62-71. 
Bergum, B. O. (1975). Self-perceptions of creativity among academic inventors and non-inventors. Perceptual and Motor Skills, 40, 78.

Bergum, B. O., \& Bergum, J. E. (1979). Creativity, perceptual stability, and self-perception. Bulletin of the Psychonomic Society, 14, 61-63.

Bergum, B. O., \& Flamm, L. O. (1975). Perceptual stability, image size, binocularity, and creativity. Perceptual and Motor Skills, 41, 667-671.

Bergum, J. E., \& Bergum, B. O. (1979). Self-perceived creativity and ambiguous figure reversal rates. Bulletin of the Psychonomic Society, 14, 373-374.

Bergum, J. E., \& Bergum, B. O. (1980) Reliability of reversal rates as a measure of perceptual stability. Perceptual and Motor Skills, 50, 1038.

Gregory, R. L. (1970). The intelligent eye. New York: McGrawHill.

Gregory, R. L. (1974). Choosing a paradigm for perception. In E. C. Carterette \& M. P. Friedman (Eds.), Handbook of perception (Vol. 1). New York: Academic Press.
Klintman, H. (1978). A comparison of figural reversal patterns with periodic changes in responsiveness in a serial naming task. Psychological Research Bulletin, 18, 1-10.

KoHLER, W. (1960). Dynamics in psychology. New York: Grove Press.

Long, G. M., \& Toppino, T. C. (1981). Multiple representations of the same reversible figure: Implications for cognitive decisional interpretations. Perception, 10, 231-234.

Mason, J., Kaszor, P., \& Bourassa, C. M. (1973). Perceptual structure of the Necker cube. Nature, 244, 54-56.

Orbach, J., Erlich, D., \& Heath, H. A. (1963). Reversibility of the Necker cube: I. An examination of the concept of "satiation of orientation." Perceptual and Motor Skills, 17, 439-458.

Smith, O. W., Imparato, N., \& Exner, J. E., JR. (1968). Effects of practice on reversals of incomplete Necker cubes. Perceptual and Motor Skills, 27, 951-954.

(Manuscript received for publication July 25, 1983.) 